\title{
SECOND ORDER SLIDING MODE OBSERVER FOR STABLE CONTROL OF A WALKING BIPED
}

\author{
V. Lebastard, Y. Aoustin, F. Plestan \\ IRCCyN, Ecole Centrale de Nantes, CNRS, Université de \\ Nantes - BP 92101, 1, rue de la Noë, 44321 Nantes cedex \\ 03, France - Vincent.Lebastard@irccyn.ec-nantes.fr
}

\begin{abstract}
An observer-based controller is proposed for the walking of a biped without feet, i.e. an underactuated system in single support phase. The originality is both: first, the observer is based on second-order sliding mode approach and is original in biped robot context. Secondly, an existing "simplified" Poincaré's sections-based analysis of the stability of the walking is adapted to nonlinear system with not fully available state variables. Copyright ${ }^{\complement}$ 2005 IFAC.
\end{abstract}

Keywords: Second order sliding mode observer, Poincaré's sections, biped.

\section{INTRODUCTION}

This work proposes an observer-based control of a biped, supposing that only relative angular positions are available. An important point of this work consists in the proof of the stability of observer-based controller and is a direct adaptation of (Grizzle et al., 2001), which has proposed a "simplified" way based on Poincaré's sections in the case where all the state is available. Usually, a precise measurement of the absolute orientation of a walking biped robot is, by a technical point-of-view, quite difficult to get. However, the generalized coordinates are often necessary for the control of the walking robot (Aoustin and Formal'sky, 2003; Chevallereau et al., 2003; Mu and $\mathrm{Wu}, 2003$; Plestan et al., 2003). Then, there is a real interest to develop observers in order to estimate absolute angular positions and velocities from only the knowledge of the relative angular variables. To our best knowledge, very few works have been done for the design of such observers, these works being done especially for the estimations of velocities (for noiseless differentiation) by supposing that all the angular variables are measured (Micheau et al., 2003). A first work on the design of observer/controller using only the measurement of joint link angular variables for a biped robot in case of its stabilization in a vertical position and its walking has been made by the authors and is based on high gain observer (Lebastard et al., 2004). In this latter work, no controller-observer superposition stability proof has been stated. In the present paper, an original observer, based on second-order sliding mode control (Levant, 1993), is used (still used for the design of observers for electrical motors (Floquet et al., 2002)) because a property of this class of observers is the finite-time convergence of the estimation error. Coupled with a finite-time convergence controller, the use of this class of observers induces an extension of (Grizzle et al., 2001) for the stability of a walking gait of a biped with not fully available state variables.

\section{MODEL OF THE BIPED}

The complete model of the biped consists of two parts: the differential equations describing the dynamics of the robot during the swing phase, and an impulse model of the contact event (the impact 
between the swing leg and the ground is modeled as a contact between two rigid bodies (Hurmuzlu and Marghitu, 1994)). The contact between the stance leg and the ground is modeled as a pivot.
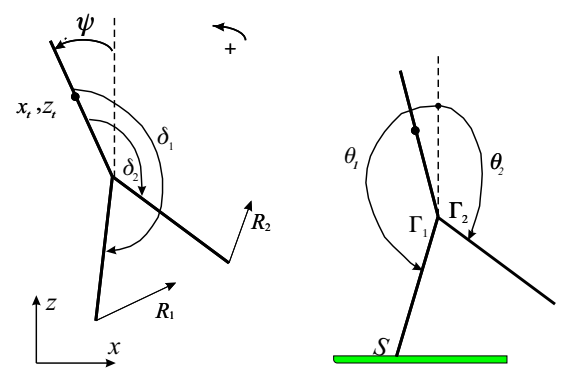

Fig. 1. Biped's diagram: generalized coordinates, torques, forces applied to the leg tips.

\subsection{Swing motion equations}

A planar three-link biped is considered (see Figure 1) and is composed by a torso and two legs without knees and feet. The joints between the torso and the legs are actuated by two actuators localized in the hips. Then, the biped is underactuated in single support. The dynamic model is given by the following Lagrange matrix equations

$$
D_{e} \ddot{q}_{e}+C_{e} \dot{q}_{e}+G_{e}=B_{e} \Gamma+D_{R} R
$$

with $q_{e}=:\left[\begin{array}{lll}q^{\prime} & x_{t} & z_{t}\end{array}\right]^{\prime}$. Vector $q$, is composed composed of the joint variables and the orientation angle of the trunk, $q:=\left[\begin{array}{lll}\delta_{1} & \delta_{2} & \psi\end{array}\right]^{\prime}$. Coordinates $\left(x_{t}, z_{t}\right)$, represents the mass center of the trunk (see Figure 1$)$. $D_{e}\left(\delta_{1}, \delta_{2}\right)$ is the $5 \times 5$ symmetric positive inertia matrix, $C_{e}(q, \dot{q})(5 \times$ $5)$ represents the Coriolis and centrifugal effects and $G_{e}\left(q_{e}\right)(5 \times 1)$ the gravity effects. $B_{e}$ is a $5 \times 2$-matrix composed of 1 and 0 and $D_{R}\left(q_{e}\right)$ is the $5 \times 2$-Jacobian matrix linking leg tips and joints. $\Gamma=\left[\begin{array}{ll}\Gamma_{1} & \Gamma_{2}\end{array}\right]^{\prime}$ are the two actuator torques. $R=\left[\begin{array}{llll}R_{N 1} & R_{T 1} & R_{N 2} & R_{T 2}\end{array}\right]^{\prime}$ represents the ground reaction acting on the stance leg tips. During the swing phase, the stance leg is acting as a pivot; the contact of the swing leg with the ground results in no rebound and no slipping of the swing leg. Then Equation (1) can be simplified and rewritten as

$$
D \ddot{q}+C \dot{q}+G=B \Gamma
$$

where $D\left(\delta_{1}, \delta_{2}\right)(3 \times 3)$ is the symmetric positive inertia matrix, $C(q, \dot{q})(3 \times 3)$ represents the Coriolis and centrifugal effects, and $G(q)(3 \times 1)$ the gravity effects. $B(3 \times 2)$ is a matrix composed of 1 and 0 . System (2) can be written as

$$
\begin{aligned}
\dot{x} & :=\left[\begin{array}{c}
\omega \\
D^{-1}(-C \omega-G+B \Gamma)
\end{array}\right] \\
& =: f(x)+g\left(q_{r e l}\right) \cdot \Gamma
\end{aligned}
$$

with $\omega:=\dot{q}, x:=\left[q^{\prime}, \omega^{\prime}\right]^{\prime}$ and $q_{r e l}:=\left[\begin{array}{ll}\delta_{1} & \delta_{2}\end{array}\right]^{\prime}$. The state space is taken such that $x \in \mathcal{X}:=\{x:=$ $\left.\left(q^{\prime}, \omega^{\prime}\right)^{\prime} \mid q \in \mathcal{M}, \omega \in \mathcal{N}\right\}$, where $\mathcal{M}=(-\pi, \pi)^{3}$ and $\mathcal{N}=\left\{\omega \in \mathbb{R}^{3}|| \omega \mid<\omega_{M}<\infty\right\}$.

\subsection{Passive impact model}

The impact occurs at the end $t=T_{I}$ of a single support phase, when the swing leg tip touches the ground. State the subscripts 2 for the swing leg and 1 for the stance leg during the single support phase. An impact occurs when angle $\delta_{2}$ equals a desired value $\delta_{2 f}$, i.e. $x \in \mathcal{S}=\left\{x \in \mathcal{X} \mid \delta_{2}=\right.$ $\left.\delta_{2 f}\right\}$. The choice of $\delta_{2 f}$ directly influences the length of the step. Assume that the impact is passive and absolutely inelastic, and the swing leg touching the ground does not slip and the previous stance leg takes off the ground. Then the angular positions are continuous, the angular velocities discontinuous. The ground reactions at the impact can be considered as impulsive forces and defined by Dirac delta-functions. Considering $x^{+}:=\left(q^{+}, \omega^{+}\right)$(state just before the impact) in terms of $x^{-}:=\left(q^{-}, \omega^{-}\right)$(state just after the impact), the impact can be written as (Grizzle et al., 2001) with following state form $x^{+}=\Delta\left(x^{-}\right)$.

\subsection{Nonlinear model all over the step}

The overall biped model can be expressed as a system with impulse effects as

$$
\begin{aligned}
\dot{x} & =f(x)+g\left(q_{\text {rel }}\right) \Gamma & x^{-}(t) \notin \mathcal{S} \\
x^{+} & =\Delta\left(x^{-}\right) & x^{-} \in \mathcal{S} .
\end{aligned}
$$

where $\mathcal{S}=\left\{x \in \mathcal{X} \mid \delta_{2}=\delta_{2 f}\right\}$.

\section{CONTROL LAW}

The control for the walking consists in maintaining the angle of the torso at some constant value $\psi_{d}$ and controlling the swing leg such that it behaves as a mirror image of the stance leg, $\theta_{2}=-\theta_{1}$ (see Figure 1) (Grizzle et al., 2001). During the single support phase, the degree of the underactuation equals one: only two outputs can be driven. Then, the robot gets a walking motion if the controller drives to zero the following outputs $y:=\left[\begin{array}{ll}y_{1} & y_{2}\end{array}\right]^{\prime}=\left[\psi-\psi_{d} \theta_{2}+\theta_{1}\right]^{\prime}=: h(x)$. As the relative degree of each output component equals 2 , one gets $\ddot{y}=L_{f}^{2} h(x)+L_{g} L_{f} h(x) \Gamma$. The control consists in decoupling the system and in imposing a desired dynamic response. Note that, in $\mathcal{X}$, the decoupling matrix $L_{g} L_{f} h$ never equals zero. Control law $u$ is then

$$
\Gamma:=\left[L_{g} L_{f} h\right]^{-1}\left[-L_{f}^{2} h+v\right]
$$


to get a linear behavior of the output vector: $\ddot{y}=v$. In the present work, control law $v$ comes from (Bhat and Bernstein, 1998):

$$
v=\Psi(y, \dot{y}):=\frac{1}{\epsilon} \cdot\left[\begin{array}{l}
\psi_{1}\left(y_{1}, \epsilon \cdot \dot{y}_{1}\right) \\
\psi_{2}\left(y_{2}, \epsilon \cdot \dot{y}_{2}\right)
\end{array}\right] .
$$

Each function $\psi_{i}\left(y_{i}, \epsilon \cdot \dot{y}_{i}\right)$, is defined as

$$
\begin{aligned}
\psi_{i}:= & -\operatorname{sign}\left(\phi_{i}\left(y_{i}, \epsilon \cdot \dot{y}_{i}\right)\right) \cdot\left|\phi_{i}\left(y_{i}, \epsilon \cdot \dot{y}_{i}\right)\right|^{\frac{\alpha}{2-\alpha}} \\
& -\operatorname{sign}\left(\epsilon \cdot \dot{y}_{i}\right) \cdot\left|\epsilon \cdot \dot{y}_{i}\right|^{\alpha}
\end{aligned}
$$

with $\phi_{i}(\cdot)=y_{i}+\frac{1}{2-\alpha} \operatorname{sign}\left(\epsilon \cdot \dot{y}_{i}\right) \cdot\left|\epsilon \cdot \dot{y}_{i}\right|^{2-\alpha}$ and $0<\alpha<1$. Real parameter $\epsilon>0$ allows the settling time of the controllers to be adjusted. Let $T_{C}$ denote the convergence time, such that $0 \leq T_{C} \leq \infty$ and $h(x)=0$ for $t \geq T_{C}$.

\section{SLIDING MODE OBSERVER}

\subsection{Preliminaries}

Consider the dynamic part of (4), with $\bar{y}:=$ $\left[\begin{array}{ll}q_{r e l}^{\prime} & \dot{q}_{r e l}^{\prime}\end{array}\right]^{\prime}=:\left[\begin{array}{lll}\bar{y}_{1} & \cdots & \bar{y}_{4}\end{array}\right]^{\prime}$ the measured variables,

$$
\dot{x}=f(x)+g\left(\bar{y}_{1}, \bar{y}_{2}\right) \Gamma, \quad \bar{y}=: h(x)
$$

with $x \in \mathcal{X}, \Gamma \in \mathbb{R}^{2}$ and $\bar{y} \in \mathbb{R}^{4}$. Let $\mathcal{O}$ denote the generic observability space ${ }^{1}$ defined by $\mathcal{O}=\tilde{\mathcal{X}} \cap(\tilde{\mathcal{Y}}+\tilde{\mathcal{U}})$, with $\tilde{\mathcal{X}}=\operatorname{Span}_{\mathcal{K}}\{\mathrm{d} x\}, \tilde{\mathcal{Y}}=$ $\operatorname{Span}_{\mathcal{K}}\left\{\mathrm{d} \bar{y}^{(j)}, j \geq 0\right\}$ and $\tilde{\mathcal{U}}=\operatorname{Span}_{\mathcal{K}}\left\{\mathrm{d} u^{(j)}, j \geq\right.$ $0\} \equiv 0 . \operatorname{Span}_{\mathcal{K}}$ is a space spanned over field $\mathcal{K}$ of meromorphic functions of $x . \bar{y}^{(w)}$ (resp. $u$ ) denotes the $w^{\text {th }}$ time derivative of $\bar{y}$ (resp. $u$ ).

Definition 1. System (8) is generically observable if $\operatorname{dim} \mathcal{O}=6$.

This condition is called Rank condition of generic observability. In fact, in $\mathcal{X}$, there exist some points for which the dimension of $\mathcal{O}$ fails: this loss of observability will be studied in a following section. Recall that all the coordinates of the state vector and in particular velocities are bounded (see definition of $\overline{\mathcal{X}}$ ).

As $g\left(\bar{y}_{1}, \bar{y}_{2}\right) \Gamma$, the input-output injection term of (8), is fully known, an observer for (8) can be designed by the following way. Consider the next nonlinear system, which is the part of (8) without the input-output injection term $(z$ has the same dimension than $x$ )

$$
\dot{z}=f(z), \quad \bar{y}=h(z)
$$

As (9) is generically observable, let $\left[k_{1} k_{2} k_{3} k_{4}\right]^{\prime}=$ $\left[\begin{array}{llll}1 & 1 & 2 & 2\end{array}\right]^{\prime}$ denote the observability indices (Krener and Respondek, 1985) of outputs $\left[\begin{array}{llll}\bar{y}_{1} & \bar{y}_{2} & \bar{y}_{3} & \bar{y}_{4}\end{array}\right]^{\prime}=$

\footnotetext{
1 If a property is generically satisfied, that means that this property is locally satisfied around a regular point $x_{0} \in \mathcal{X}$.
}

$\left[\begin{array}{llll}\delta_{1} & \delta_{2} & \dot{\delta}_{1} & \dot{\delta}_{2}\end{array}\right]^{\prime}$ such that $\zeta:=\left[\begin{array}{llllll}\bar{y}_{1} & \bar{y}_{2} & \bar{y}_{3} & \dot{\bar{y}}_{3} & \bar{y}_{4} & \dot{\bar{y}}_{4}\end{array}\right]^{\prime}=$ : $\Psi(z)$ is locally invertible, and then defines a local state coordinates transformation. Nonlinear system (9) can be written as

$$
\begin{aligned}
\dot{\zeta} & =\left[\begin{array}{llllll}
0 & 0 & 1 & 0 & 0 & 0 \\
0 & 0 & 0 & 0 & 1 & 0 \\
0 & 0 & 0 & 1 & 0 & 0 \\
0 & 0 & 0 & 0 & 0 & 0 \\
0 & 0 & 0 & 0 & 0 & 1 \\
0 & 0 & 0 & 0 & 0 & 0
\end{array}\right] \\
A & \underbrace{\left[\begin{array}{llllll}
1 & 0 & 0 & 0 & 0 & 0 \\
0 & 1 & 0 & 0 & 0 & 0 \\
0 & 0 & 1 & 0 & 0 & 0 \\
0 & 0 & 0 & 0 & 1 & 0
\end{array}\right]}_{A} \underbrace{\left[\begin{array}{c}
0 \\
0 \\
0 \\
\Phi_{4}(\zeta) \\
0 \\
\Phi_{6}(\zeta)
\end{array}\right]}_{\Phi(\zeta)} \\
\bar{y} & =\underbrace{(\zeta)}
\end{aligned}
$$

Suppose that there exists a system defined as

$$
\dot{\hat{\zeta}}=A \hat{\zeta}+\Phi(\hat{\zeta})+\chi(\hat{\zeta}, \bar{y})
$$

which is an observer of (10). Then, it is easily shown that the system

$$
\dot{\hat{x}}=f(\hat{x})+g\left(\bar{y}_{1}, \bar{y}_{2}\right) \Gamma+\left[\frac{\partial \Psi(\hat{x})}{\partial \hat{x}}\right]^{-1} \chi(\hat{\zeta}, \bar{y})
$$

is an observer of (8).

\subsection{Second-order sliding mode observer}

Let $\chi(\hat{\zeta}, \bar{y})$ denote by $\chi=\left[\begin{array}{lll}\chi_{1} & \cdots & \chi_{6}\end{array}\right]^{\prime}$. One uses the standard sliding mode approach for $\chi_{1}$ and $\chi_{2}$ by $\chi_{1}=-\lambda_{1} \operatorname{sign}\left(\hat{\zeta}_{1}-\zeta_{1}\right)$ and $\chi_{2}=-\lambda_{2} \operatorname{sign}\left(\hat{\zeta}_{2}-\right.$ $\left.\zeta_{2}\right)$. Then, from (11) and (10), the dynamics of $e_{1}$ and $e_{2}$ read as (with $e_{i}=\hat{\zeta}_{i}-\zeta_{i}, 1 \leq i \leq 6$ ) $\dot{e}_{1}=e_{3}-\lambda_{1} \operatorname{sign}\left(e_{1}\right)$ and $\dot{e}_{2}=e_{5}-\lambda_{2} \operatorname{sign}\left(e_{2}\right)$. Then, $e_{1}$ and $e_{2}$ converge in finite time towards zero if the sliding condition $\dot{e}_{i} e_{i}<0(1 \leq i \leq 2)$ is fulfilled, i.e.

$$
\begin{aligned}
& \lambda_{1}>\operatorname{Max}\left|e_{3}\right|=\operatorname{Max}\left(\left|\dot{\hat{\delta}}_{1}-\dot{\delta}_{1}\right|\right) \\
& \lambda_{2}>\operatorname{Max}\left|e_{5}\right|=\operatorname{Max}\left(\left|\dot{\hat{\delta}}_{2}-\dot{\delta}_{2}\right|\right)
\end{aligned}
$$

Functions $\chi_{i}$, are defined such that the estimation errors $e_{i}(i=3, \ldots, 6)$ converge towards zero in finite-time and read as

$$
\begin{array}{ll}
\chi_{3}=0 & \chi_{4}=-\Lambda_{3} \operatorname{sign}\left(e_{3}\right) \\
\chi_{5}=0 & \chi_{6}=-\Lambda_{5} \operatorname{sign}\left(e_{5}\right)
\end{array}
$$

Given that $\dot{e}_{3}=e_{4}$ and $\dot{e}_{5}=e_{6}$, one gets

$$
\begin{aligned}
& \ddot{e}_{3}=\Phi_{4}(\hat{\zeta})-\Phi_{4}(\zeta)-\Lambda_{3} \operatorname{sign}\left(e_{3}\right) \\
& \ddot{e}_{5}=\Phi_{6}(\hat{\zeta})-\Phi_{6}(\zeta)-\Lambda_{5} \operatorname{sign}\left(e_{5}\right)
\end{aligned}
$$

The choice for $\Lambda_{3}$ and $\Lambda_{5}$, based on the twisting algorithm (Levant, 1993), allows to ensure that the 
previous system converges to zero in finite-time, and then to ensure that $e_{3}, e_{4}, e_{5}, e_{6}$ reach zero. The twisting algorithm ensures this convergence if (Levant, 1993)(Floquet et al., 2002) (for $i=$ $\{3,5\})$

$$
\begin{aligned}
& \Lambda_{i}= \begin{cases}\lambda_{m_{i}} & \text { if } \quad e_{i} e_{i+1} \leq 0 \\
\lambda_{M_{i}} & \text { if } \quad e_{i} e_{i+1}>0\end{cases} \\
& \lambda_{m_{i}}>\operatorname{Max}\left(\left|\Phi_{i+1}(\hat{\zeta})-\Phi_{i+1}(\zeta)\right|\right) \\
& \lambda_{M_{i}}>3 \lambda_{m_{i}}
\end{aligned}
$$

Then, a second-order sliding mode observer for (8) reads as

$$
\begin{aligned}
& \dot{\hat{x}}=f(\hat{x})+g\left(\bar{y}_{1}, \bar{y}_{2}\right) \Gamma \\
& +\left[\frac{\partial \Psi(\hat{x})}{\partial \hat{x}}\right]^{-1}\left[\begin{array}{c}
-\lambda_{1} \operatorname{sign}\left(\hat{\delta}_{1}-\delta_{1}\right) \\
-\lambda_{2} \operatorname{sign}\left(\hat{\delta}_{2}-\delta_{2}\right) \\
0 \\
-\Lambda_{3} \operatorname{sign}\left(\dot{\hat{\delta}}_{1}-\dot{\delta}_{1}\right) \\
0 \\
-\Lambda_{5} \operatorname{sign}\left(\dot{\hat{\delta}}_{2}-\dot{\delta}_{2}\right)
\end{array}\right]
\end{aligned}
$$

Let $T_{O}$ denote the observer convergence time, such that $0 \leq T_{O} \leq \infty$ and $x=\hat{x}$ for $t \geq T_{O}$.

\subsection{Loss of observability}

During the swing phase, and along the desired trajectories, there is loss of observability, which implies that the determinant of $\frac{\partial \Psi(\hat{x})}{\partial \hat{x}}$ crosses zero (Figure 2). It induces a problem for the design of the observer (17). Around this singular point, it is necessary to make adaptations/corrections of the observer, which is not valid at exactly the singularity. Two intuitive and quite natural solutions are used:

Case 1. The observer is turned into an estimator

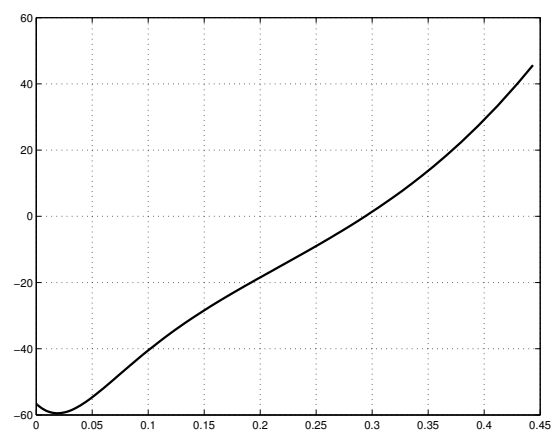

Fig. 2. $\operatorname{det}\left(\frac{\partial \Psi(x)}{\partial x}\right)$ versus time (sec.) along one step when $\left|\operatorname{det}\left(\frac{\mathrm{d} \Psi(\hat{\mathrm{x}})}{\mathrm{d} \hat{\mathrm{x}}}\right)\right|<\mathrm{D}_{\min }$, with $D_{\min }$ a positive real arbitrarily stated by the user. If the observer has not still converged when the singularity appears, there is a discontinuity on observer dynamics. In the opposite case, there is no discontinuity viewed that the corrective term of the observer still equals zero. Note that, viewed that the observer is finite-time convergence one, it is possible to tune the observer gain such that the observer convergence time is smaller than the singularity moment.

Case 2. To avoid the discontinuity appearing in the previous solution, a smooth corrective term is added at the observer such that

$$
\dot{\hat{x}}=f(\hat{x})+g\left(\bar{y}_{1}, \bar{y}_{2}\right) \Gamma+\Theta \chi(\hat{\zeta}, \bar{y})
$$

with

$$
\Theta=\left\{\begin{array}{l}
{\left[\frac{\mathrm{d} \Psi(\hat{x})}{\mathrm{d} \hat{x}}\right]^{-1}} \\
\text { if }\left|\operatorname{det}\left(\frac{\mathrm{d} \Psi(\hat{x})}{\mathrm{d} \hat{x}}\right)\right| \geq D_{\text {min }} \\
\frac{\mid\left[\frac{\mathrm{d} \Psi(\hat{x})}{\mathrm{d} \hat{x}}\right]}{D_{\min }}\left[\frac{\mathrm{d} \Psi(\hat{x})}{\mathrm{d} \hat{x}}\right]^{-1} \\
\text { if } 0<\left|\operatorname{det}\left(\frac{\mathrm{d} \Psi(\hat{x})}{\mathrm{d} \hat{x}}\right)\right|<D_{\text {min }} \\
0 \text { if }\left|\operatorname{det}\left(\frac{\mathrm{d} \Psi(\hat{x})}{\mathrm{d} \hat{x}}\right)\right|=0
\end{array}\right.
$$

Of course, this multiplication acts on observer gain values and implies that, around the singular point, the convergence conditions are not satisfied. It means that the singularity area must be "sufficiently small".

\section{SIMULATIONS}

The control law described in Section 3 is applied with parameters $\alpha=0.9$ and $\epsilon=20$. The initial real and estimated values have been respectively stated as $\left[\begin{array}{llllll}\delta_{1} & \delta_{2} & \psi & \dot{\delta}_{1} & \dot{\delta}_{2} & \dot{\psi}\end{array}\right]=\left[1.1 \hat{\delta}_{1}(0)\right.$ $\left.1.1 \hat{\delta}_{2}(0) 1.15 \hat{\psi}(0) 1.2 \dot{\hat{\delta}}_{1}(0) 1.2 \dot{\hat{\delta}}_{2}(0) \psi \dot{\hat{\hat{\psi}}(0)}\right],\left[\hat{\delta}_{1} \hat{\delta}_{2}\right.$

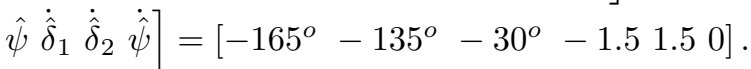
Parameters $D_{\min }$ has been stated to 5 , and the sliding mode observer parameters are $\lambda_{1}=1$, $\lambda_{2}=1, \lambda_{m_{3}}=10, \lambda_{M_{3}}=50, \lambda_{m_{5}}=10$, $\lambda_{M_{5}}=50$. The choice of observer and control law parameters has been made with respect to closedloop dynamics and admissible maximum value for input (saturation). Figure 3 displays absolute position $\psi$, estimated position $\hat{\psi}$ and estimation error $\psi-\hat{\psi}$. Figure 4 displays absolute velocity $\dot{\psi}$, estimate velocity $\dot{\hat{\psi}}$ and estimation error $\dot{\psi}-\dot{\hat{\psi}}$. Figure 5 displays walking over several steps.

\section{STABILITY}

The purpose of this section is to prove the asymptotic stability or instability of trajectories resulting from the biped in closed loop with the controller (5) coupled with the observer (18). An important result from (Grizzle et al., 2001) is 

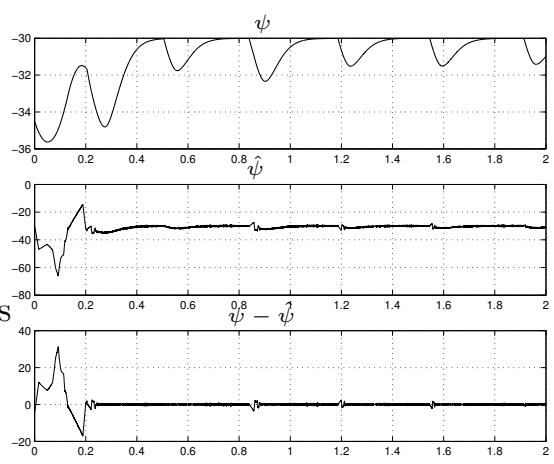

Fig. 3. From top to bottom, absolute angle $\psi$; estimated absolute angle $\hat{\psi}$ and estimation error $\psi-\hat{\psi}$ (rad.) versus time (sec.)
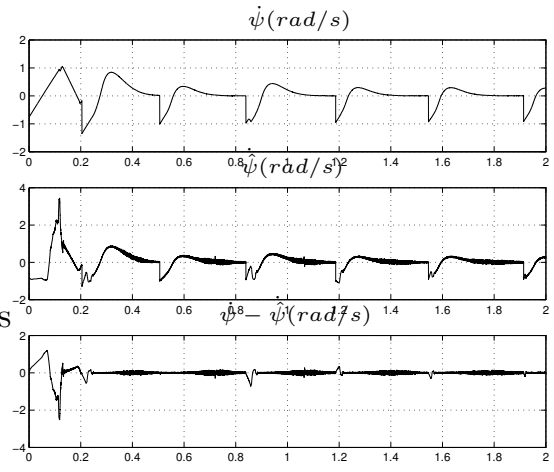

Fig. 4. From top to bottom, absolute velocity $\dot{\psi}$; estimated absolute velocity $\dot{\hat{\psi}}$ and estimation error $\dot{\psi}-\dot{\hat{\psi}}(\mathrm{rad} / \mathrm{s})$ versus time (sec.)

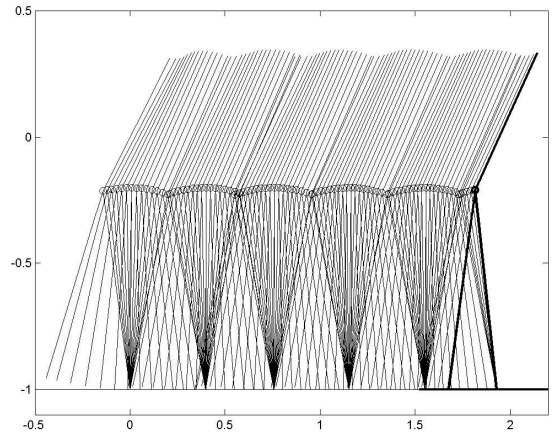

Fig. 5. Plot of walking as a sequence of stick figure.

that stability (or instability) can be proven on the basis of the restriction of the Poincaré map to a one-dimensional manifold. This section proposed a first extension of this latter result to observer-based controlled systems. As the "real" state vector is not fully measured, the "real" zeros dynamics and impact surface manifolds can not be used in the stability proof. The idea is then to suppose that the estimated state is on "estimated" zeros dynamics and impact surface manifolds. As the observer and controller have finite-time convergence, at the end of the first step, it is sure that the estimated manifolds are the same than "real" ones. Then, it is possible to use the standard reduced Poincaré's approach to establish, over the second step, the stability.

Let $\hat{Z}$ denote the "estimated" zero dynamics manifold, $\hat{Z}=\left\{\hat{x} \in \hat{\mathcal{X}} \mid h(\hat{x})=0, L_{f} h(\hat{x})=0\right\}$, $\hat{\mathcal{S}}$ the "estimated" impact surface manifold, $\hat{\mathcal{S}}=$ $\left\{\hat{x} \in \mathcal{X} \mid \hat{\delta}_{2}=\delta_{2 f}\right\}, \hat{\mathcal{X}}:=\left\{\hat{x}:=\left(\hat{q}^{\prime}, \hat{\omega}^{\prime}\right)^{\prime} \mid \hat{q} \in\right.$ $\mathcal{M}, \hat{\omega} \in \mathcal{N}\}, \hat{q}$ and $\hat{\omega}$ being the estimated values of $q$ and $\omega$. One supposes that: The observer and the controller have been tuned such that $T_{O} \leq T_{C}<\infty$, i.e. the observer converges faster than the controller. The conditions required to define the restricted Poincaré map are (Plestan et al., 2003):

1. $\hat{\mathcal{S}} \cap \hat{Z}$ is a smooth submanifold of $\hat{\mathcal{X}}$. It is equivalent to the fact that the map $\left[\begin{array}{c}h(\hat{x}) \\ L_{f} h(\hat{x}) \\ \hat{\delta}_{2}\end{array}\right]=$ $\left[\begin{array}{c}\hat{\psi}-\psi_{d} \\ \hat{\theta}_{1}+\hat{\theta}_{2} \\ \dot{\hat{\psi}} \\ \hat{\omega}_{1}+\hat{\omega}_{2} \\ \hat{\delta}_{2}\end{array}\right]$

has constant rank equal to 5 on $\hat{\mathcal{S}} \cap$

$\hat{Z}$, which is obvious to prove. If $(\hat{q}, \hat{\omega}) \in \hat{\mathcal{S}} \cap$ $\hat{Z}, \hat{q}$ equals a constant, denoted $\hat{q}_{0}$. Let $\gamma:=$ $\left[h(\hat{x})^{\prime} \hat{\theta}_{1}(\hat{x})\right]^{\prime}$ which has full rank at $\hat{q}_{0}$. On $\hat{Z}$, one has $\dot{h}(\hat{x})=0$ and (with $\hat{\omega}_{1}:=\dot{\hat{\theta}}_{1}$ ) $\left[\begin{array}{c}0 \\ \hat{\omega}_{1}\end{array}\right]=\frac{\partial \gamma}{\partial \hat{q}} \hat{\omega}$ Thus, $p: \mathbb{R} \rightarrow \hat{\mathcal{S}} \cap \hat{Z}$ defined by $p\left(\hat{\omega}_{1}\right)=\left[\left[\frac{\partial \gamma\left(\hat{q}_{0}\right)}{\partial \hat{q}}\right]^{-1} \hat{\omega}_{1}\right]$ is a diffeomorphism from $\mathbb{R}$ to $\hat{S} \cap \hat{Z}$.

2. As shown in (Grizzle et al., 2001), the decoupling matrix $L_{g} L_{f} h(\hat{x})$ is invertible on $\hat{\mathcal{X}}$.

3. The cross section for the Poincaré map will be taken to be $\hat{S}$, the "estimated" impact surface. Define $\lambda: \mathbb{R} \rightarrow \mathbb{R}$ computed by the following manner

- Let $\hat{\omega}_{1}^{-}(0)>0$ denote the initial estimated angular velocity just before the first impact. Compute $\hat{x}^{-}(0):=p\left(\hat{\omega}_{1}^{-}(0)\right)$, the estimated position of the robot before the impact. State the real state before the impact as (given that $\delta_{1}, \delta_{2}$ and the corresponding velocities are measured) $x^{-}(0):=$ $\left[\hat{\delta}_{1}^{-}(0) \hat{\delta}_{2}^{-}(0) \psi^{-}(0) \dot{\hat{\delta}}_{1}^{-}(0) \dot{\hat{\delta}}_{2}^{-}(0) \dot{\psi}^{-}(0)\right]^{\prime} \circ \mathrm{Ap}-$ ply the impact model to $\hat{x}^{-}(0)$ (resp. $\left.x^{-}(0)\right)$, $\hat{x}^{+}(0)=\Delta\left(\hat{x}^{-}(0)\right)\left(\right.$ resp. $\left.x^{+}(0)=\Delta\left(x^{-}(0)\right)\right)$.

- Use $x^{+}(0)$ as the initial condition in (4) controlled by $(6)$ which uses $\hat{x}$. Simulate until one of the following happens

a) There exists a time $T_{I}^{1}$ for which $\hat{\delta}_{2}=\delta_{2 f}$ and $T_{O} \leq T_{C}^{1}<T_{I}^{1}<\infty^{2}$, then apply again the impact model to $\hat{x}^{-}\left(T_{I}^{1}\right), \hat{x}^{+}\left(T_{I}^{1}\right)=\Delta\left(\hat{x}^{-}\left(T_{I}^{1}\right)\right)^{3}$. Use $\hat{x}^{+}\left(T_{I}^{1}\right)$ as the initial condition in (4) con-

\footnotetext{
${ }^{2} T_{C}^{1}$ is the controller convergence time during the first step.

3 At this time, the real and estimated state variables have same values, viewed that the observer has finite-time
} 
trolled by (6). If there exists a time $T_{I}^{2}$ for which $\hat{\delta}_{2}=\delta_{2}=\delta_{2 f}$ such that $T_{O} \leq T_{C}^{2}<T_{I}^{2}<\infty^{4}$, then $\lambda\left[\hat{\omega}_{1}^{-}\left(T_{I}^{1}\right)\right]:=\hat{\omega}_{1}^{-}\left(T_{I}^{2}\right)$; else $\lambda\left[\hat{\omega}_{1}^{-}\left(T_{I}^{1}\right)\right]$ is undefined at this point.

b) There does not exist a $T_{I}^{2}>0$ such that $\hat{\delta}_{2}=$ $\delta_{2 f}$; in this case, it is also true that $\lambda\left[\hat{\omega}_{1}^{-}\left(T_{I}^{1}\right)\right]$ is undefined at this point.

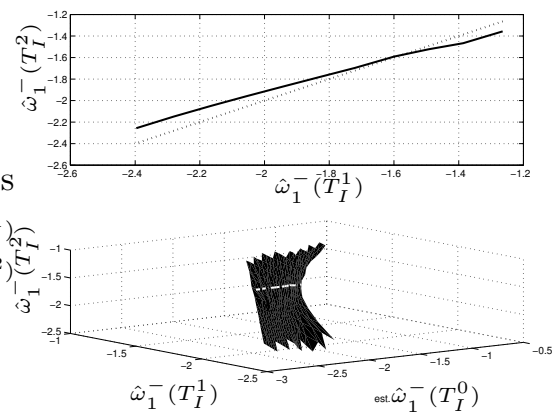

Fig. 6. Top: Function $\lambda$ (bold line) and identity function (dotted line) versus $\hat{\omega}_{1}^{-}\left(T_{I}^{1}\right)$. This graph describes the existence of an asymptotically stable walking motion. Bottom: Function $\lambda$ versus $\hat{\omega}_{1}^{-}\left(T_{I}^{1}\right)$ and $\hat{\omega}_{1}^{-}(0)$, with estimation errors on $\psi(0)$. The white segment corresponds to the identity function.

To determine if the closed-loop system (controller+observer) is stable, function $\lambda$ is computed with $\hat{\omega}_{1}^{-}(0) \in[0.5,3]$. Figure 6 -Top displays function $\lambda$ with the previous choice for $\hat{\omega}_{1}^{-}(0)$, $\psi(0)=0.8 \psi_{d}$ and $\dot{\psi}(0)=0.1$. One deduces that $\lambda$ is undefined for $\hat{\omega}_{1}^{-}\left(T_{I}^{1}\right)$ less than $-2.4 \mathrm{rad} / \mathrm{s}$ (corresponding to $\hat{\omega}_{1}^{-}(0)$ less than $-2.9 \mathrm{rad} / \mathrm{s}$ ) and more than $-1.3 \mathrm{rad} / \mathrm{s}$ (corresponding to $\hat{\omega}_{1}^{-}(0)$ more than $-1.6 \mathrm{rad} / \mathrm{s})$. A fixed point appears at approximately $\hat{\omega}_{1}^{-}\left(T_{I}^{1}\right)=-1.63 \mathrm{rad} / \mathrm{s}$, and corresponds to an asymptotically stable walking cycle. The latter cycle can be lighted up in a more general case (see Figure 6-Bottom): the 3D graph is obtained by considering the same variations for $\hat{\omega}_{1}^{-}(0)$ and same value for $\dot{\psi}(0)$, but estimation errors for unmeasured variable $\psi(0) \in\left[0.8 \psi_{d}, 1.2 \psi_{d}\right]$ and shows (with identity $3 \mathrm{D}$-representation) that the limit cycle for $\hat{\omega}_{1}^{-}\left(T_{I}^{1}\right)=-1.63 \mathrm{rad} / \mathrm{s}$ exists, in spite of different estimation errors.

\section{CONCLUSION}

With initial estimation errors on the unmeasured absolute orientation of a biped, the association of a finite-time convergence observer with a finitetime convergence control law leads to a stable cyclic gait. The existence of attraction basin is lighted up with Poincaré's map. Our objectives

\footnotetext{
convergence of estimation error, and that the observer gain have been tuned such that $T_{O}<T_{I}^{1}$.

${ }^{4} T_{C}^{2}$ is the controller convergence time during the second step.
}

are to define the attraction basin in function of unilateral constraints and to extend this work to a five link biped.

\section{REFERENCES}

Aoustin, Y. and A.M. Formal'sky (2003). Control design for a biped: Reference trajectory based on driven angles as functions of the undriven angle. Journal of Computer and Systems Sciences International 42(4), 159-176.

Bhat, S.P. and D.S. Bernstein (1998). Continuous finite-time stabilization of the translational and rotationnal double integrator. IEEE Transaction on Automatic Control 43(5), 678-682.

Chevallereau, C., G. Abba, Y. Aoustin, F. Plestan and E.R. Westervelt, C. Canudas de Wit and J.W. Grizzle (2003). Rabbit: a testbed for advanced control theory. IEEE Control Systems Magazine 23(5), 57-79.

Floquet, T., J.P. Barbot and W. Perruquetti (2002). A finite time observer for flux estimation in the induction machine. In: Proc. IEEE Conference on Control Applications. Glasgow, Scotland.

Grizzle, J.W., G. Abba and F. Plestan (2001). Asymptotically stable walking for biped robots : analysis via systems with impulse effects. IEEE Transactions on Automatic Control 46(1), 51-64.

Hurmuzlu, Y. and D.B. Marghitu (1994). Rigid body collisions of planar kinematic chains with multiple contact points. The International Journal of Robotics Research 13(1), 82-92.

Krener, A.J. and W. Respondek (1985). Nonlinear observers with linearizable error dynamics. SIAM J. Contr. Optim.

Lebastard, V., Y. Aoustin and F. Plestan (2004). Observer-based control of a biped robot. In: Proc. Fourth International Workshop on Robot Motion and Control. Puszczykowo, Poland. pp. 67-72.

Levant, A. (1993). Sliding order and sliding accuracy in sliding mode control. International Journal of Control 58(6), 1247-1263.

Micheau, P., M.A. Roux and P. Bourassa (2003). Self-tuned trajectory control of a biped walking robot. In: Proc. International Conference on Climbing and Walking Robot. Catania, Italy. pp. 527-534.

$\mathrm{Mu}, \mathrm{X}$. and Q. Wu (2003). Synthesis of a complete sagittal gait cycle for a five-link biped robot. Robotica 21, 581-587.

Plestan, F., J.W. Grizzle, E.R. Westervelt and G. Abba (2003). Stable walking of a 7-dof biped robot. IEEE Transactions on Robotics and Automation 19(4), 653-668. 